\title{
BRANQUEAMENTO DE ÓLEO DE FARELO DE ARROZ COM TERRA ATIVADA E QUITOSANA
}

\author{
C. P. PINHEIRO ${ }^{1}$, V. S. de BORBA ${ }^{1}$, R. S. POHNDORF ${ }^{1}$ e L. A. de A. PINTO ${ }^{1}$ \\ ${ }^{1}$ Universidade Federal do Rio Grande, Escola de Química e Alimentos \\ E-mail para contato: dqmpinto@furg.br
}

\begin{abstract}
RESUMO - Diferentes concentrações e tipos de adsorventes (terra ativada e quitosana) foram testados na adsorção de carotenóides e peróxidos no branqueamento de óleo de farelo de arroz (OFA). O estudo cinético do branqueamento demonstrou que utilizando $1 \%(\mathrm{~m} / \mathrm{m})$ de adsorvente durante 20 min ocorreu uma redução acentuada no teor de carotenóides e peróxidos. O branqueamento com $2 \%(\mathrm{~m} / \mathrm{m})$ de quitosana promoveu uma redução de quase $40 \%$ no índice peróxidos. O aumento da concentração de terra ativada promoveu o aumento na constante da taxa de adsorção, indicando maior rapidez no processo de branqueamento. O modelo de pseudo-segunda ordem apresentou melhor ajuste aos dados experimentais no branqueamento do óleo com terra ativada. Entretanto, o modelo de pseudo-primeira ordem foi mais adequado para descrever o comportamento cinético da adsorção com quitosana.
\end{abstract}

\section{INTRODUÇÃ̃O}

A produção de arroz branco envolve a retirada das camadas externas do grão, gerando um subproduto (farelo) que representa em torno de $8-11 \%$ do grão e contém até $25 \%$ de lipídios. O óleo de farelo de arroz (OFA) apresenta teor de ácidos graxos insaturados superior a $75 \%$, favorecendo a rancidez oxidativa e lipolítica do óleo. As etapas de refino de OFA vegetais comumente incluem degomagem, neutralização, branqueamento, deceramento e desodorização (Pestana-Bauer et al., 2012). Adsorção é uma operação unitária aplicada na etapa de branqueamento de óleos para remoção de pigmentos e produtos de oxidação. Porém, traços de ácidos graxos livres, fosfolipídios, sabão e metais também são removidos. Através de forças de interação entre o adsorvente e o adsorbato é realizada a purificação o óleo.

A quitosana é um biopolímero obtido a partir da desacetilação da quitina e apresenta potencial para adsorção de pigmentos e metais (Wan Ngah et al., 2011). De acordo com Sathivel e Prinyawiwatkul (2004), a quitosana apresenta elevada capacidade de adsorção de ácidos graxos livres em óleo de pescado. Porém, poucos estudos relatam a utilização de quitosana na etapa de branqueamento de óleos vegetais.

O objetivo deste estudo foi investigar o comportamento cinético da adsorção de carotenóides e produtos de oxidação (peróxidos) na etapa de branqueamento de OFA, utilizando diferentes concentrações e tipos de adsorventes (terra ativada e quitosana). Para interpretar o comportamento cinético os modelos de pseudo-primeira ordem e pseudosegunda ordem foram ajustados aos dados experimentais. 


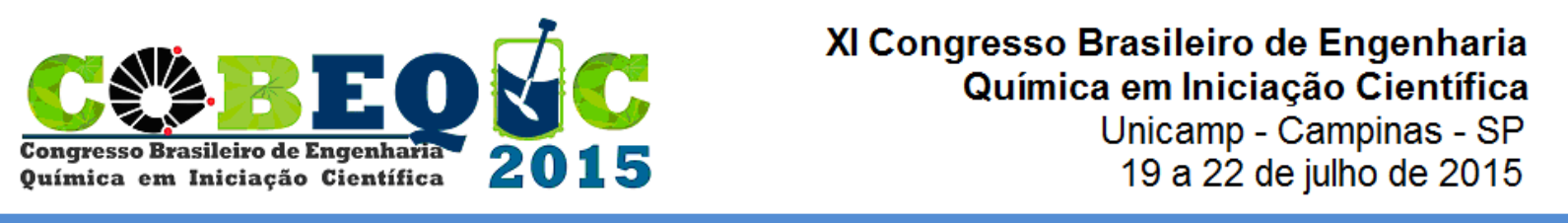

\section{MATERIAIS E MÉTODOS}

\subsection{Materiais}

O óleo de farelo de arroz neutralizado foi obtido em uma empresa de refino de óleo local e acondicionado à $-20^{\circ} \mathrm{C}$, para evitar oxidação. Os experimentos foram realizados no Laboratório de Operações Unitárias da Universidade Federal do Rio Grande (FURG), localizada no sul do Brasil. Foi utilizada terra ativada (Tonsil Supreme $110 \mathrm{FF}$ ) como adsorvente comercial nos ensaios de branqueamento. A quitosana (grau de desacetilação $85 \%$ ) foi obtida de resíduos de camarão (Penaeus brasiliensis) de acordo com o procedimento descrito por Moura et al. (2011).

\subsection{Determinação de carotenóides e produtos de oxidação}

A determinação dos carotenóides foi realizada por espectrofotômetro (Quimis, Q108, Brasil). As amostras de OFA foram diluídas em hexano $(10 \% \mathrm{~m} / \mathrm{v})$ e filtradas para evitar a influência da turbidez. O teor de carotenóides foi obtido por leituras de absorbância a $446 \mathrm{~nm}$ e expresso em mg kg-1, de acordo com metodologia proposta por MPOB Test Method (2005). Os produtos de oxidação primária foram determinados através do índice de peróxidos de acordo com o método Cd 8-53 da AOCS (1998), e expresso em mmol kg-1 óleo.

\subsection{Experimentos de adsorção}

Amostras de $40 \mathrm{~g}$ de OFA neutralizado foram aquecidas a $120 \pm 1{ }^{\circ} \mathrm{C}$ sob agitação constante $(40 \mathrm{rpm})$, utilizando um agitador magnético com aquecimento. A taxa de aquecimento foi de $7{ }^{\circ} \mathrm{C} \mathrm{min}{ }^{-1}$ e o óleo foi mantido à pressão absoluta de $40 \mathrm{mmHg}$. Após atingir a temperatura, o adsorvente (terra ativada ou quitosana) foi adicionado ao óleo em diferentes concentrações $(1 \mathrm{e} 2 \% \mathrm{~m} / \mathrm{m})$. Alíquotas foram retiradas ao longo dos $60 \mathrm{~min}$ de experimento para obtenção das curvas cinéticas. O adsorvente foi separado imediatamente do óleo por centrifugação $(4000 \times g$ por $5 \mathrm{~min})$. Os experimentos foram realizados em triplicata. As amostras foram analisadas quanto ao teor de carotenóides e índice de peróxidos. A capacidade de adsorção $\left(q_{t}\right)$ de carotenóides e peróxidos em cada tempo $t$ foi determinada pela Equação 1:

$$
q_{t}=\frac{M_{o}\left(C_{i}-C_{t}\right)}{M_{a}}
$$

onde $M_{o}$ é a quantidade de óleo (g), $C_{i}$ é a concentração inicial do adsorbato $\left(\mathrm{mg} \mathrm{kg}^{-1}\right), C_{t}$ é a concentração do adsorbato no tempo $t\left(\mathrm{mg} \mathrm{kg}^{-1}\right)$ e $M_{a}$ é a quantidade de adsorvente $(\mathrm{g})$.

\subsection{Modelos cinéticos}

O comportamento cinético da sorção de pigmentos e peróxidos foi obtido por ajuste dos modelos de pseudo-primeira ordem (Equação 2) e pseudo-segunda ordem (Equação 3). Estes modelos são baseados na capacidade de adsorção ao invés da concentração da solução (Silva et al., 2013). 


$$
\begin{aligned}
& q_{t}=q_{1}\left(1-\exp \left(-k_{1} t\right)\right) \\
& q_{t}=\frac{t}{\left(1 / k_{2} q_{2}\right)^{2}+\left(t / q_{2}\right)}
\end{aligned}
$$

onde $k_{1}$ e $k_{2}$ são as constantes da taxa de adsorção para os modelos de pseudo-primeira ordem $\left(\mathrm{min}^{-1}\right)$ e pseudo-segunda ordem $\left(\mathrm{kg} \mathrm{mg}^{-1} \mathrm{~min}^{-1}\right)$ respectivamente, e $q_{1}$ e $q_{2}$ representam a capacidade máxima de adsorção teórica $\left(\mathrm{mg} \mathrm{kg}^{-1}\right)$ no tempo $t$.

\subsection{Análise estatística}

Os modelos cinéticos foram ajustados aos dados experimentais através de regressão não linear usando o método Quasi-Newton. A qualidade do ajuste foi obtida pelo coeficiente de determinação $\left(\mathrm{R}^{2}\right)$, coeficiente de determinação ajustado $\left(\mathrm{R}_{\text {aju. }}^{2}\right)$ e erro médio relativo (EMR). Os parâmetros foram calculados (95\% de confiança) pelo software Statistic 7.0 (StatSoft, USA) e os gráfico construídos pelo software SigmaPlot 10.0 (Systat, USA).

\section{RESULTADOS E DISCUSSÃO}

\subsection{Adsorção de pigmentos}

O comportamento cinético da adsorção de carotenóides de OFA é apresentado na Figura 1. O teor de carotenóides do óleo neutralizado foi de $12,47 \pm 0,18 \mathrm{mg} \mathrm{kg}^{-1}$. Este valor diminuiu com o tempo de processo, independentemente das condições estudadas. Diferenças podem ser observadas entre a concentração do adsorvente e especialmente entre os tipos de adsorventes. $\mathrm{O}$ aumento da concentração de adsorvente tende a acelerar o processo de adsorção. Após 20 min de branqueamento com terra ativada, poucas variações no teor de carotenóides são observadas para ambas as concentrações de adsorventes, indicando uma tendência ao equilíbrio do sistema. Entretanto, para a quitosana esta tendência foi observada somente após 30 min de branqueamento.

Figura 1 - Curvas cinéticas da adsorção de carotenóides por $(\bullet)$ terra ativada e $(\circ)$ quitosana nas concentrações de adsorvente (A) $1 \%$ e (B) $2 \%$
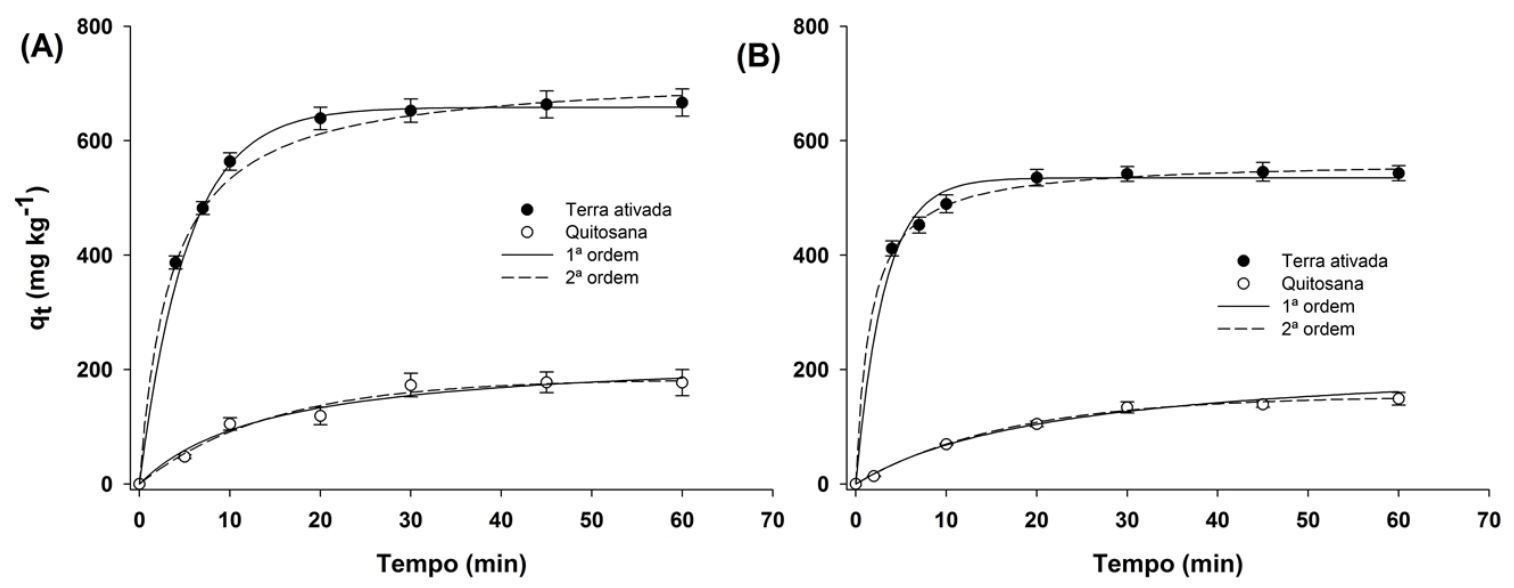
Utilizando $1 \%$ de terra ativada e 20 min de branqueamento a capacidade de adsorção foi de $660,00 \pm 19,56 \mathrm{mg}_{\text {carotenóides }} \mathrm{kg}^{-1}$ terra ativada (Figura 1A). Neste mesmo tempo, quando se

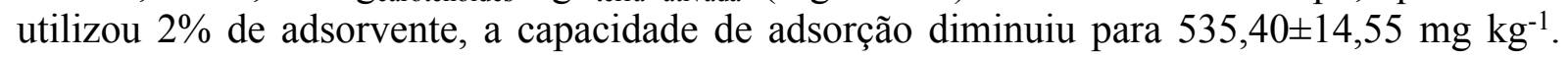
Esta diminuição ocorreu devido ao aumento da quantidade de terra ativada em relação aos carotenóides disponíveis para serem adsorvidos. Assim, a terra ativada proporcionou uma redução de 82,96\% no teor de carotenóides após 20 min de branqueamento utilizando $2 \%$ de adsorvente. Estes valores estão coerentes com a literatura. Worasith et al. (2011) relatou uma redução de $81,93 \%$ na cor do óleo de farelo de arroz utilizando adsorvente comercial em condições de processo semelhantes as deste trabalho.

\subsection{Adsorção de peróxidos}

A quitosana foi eficiente na remoção de produtos de oxidação primária, embora com capacidade de adsorção menor do que a terra ativada (Figura 2). A utilização de $2 \%$ de quitosana reduziu aproximadamente $40 \%$ dos peróxidos em $30 \mathrm{~min}$ de branqueamento, tendendo ao equilíbrio. Terras branqueadoras ativadas adsorvem preferencialmente compostos polares e são capazes de reduzir praticamente a zero os produtos de oxidação primária. Porém, segundo Silva et al. (2014) durante o branqueamento pode ocorrer a formação de compostos de oxidação secundária que serão removidos na desodorização.

Figura 2 - Curvas cinéticas da adsorção de peróxidos por $(\bullet)$ terra ativada e (०) quitosana nas concentrações de adsorvente (A) $1 \%$ e (B) $2 \%$
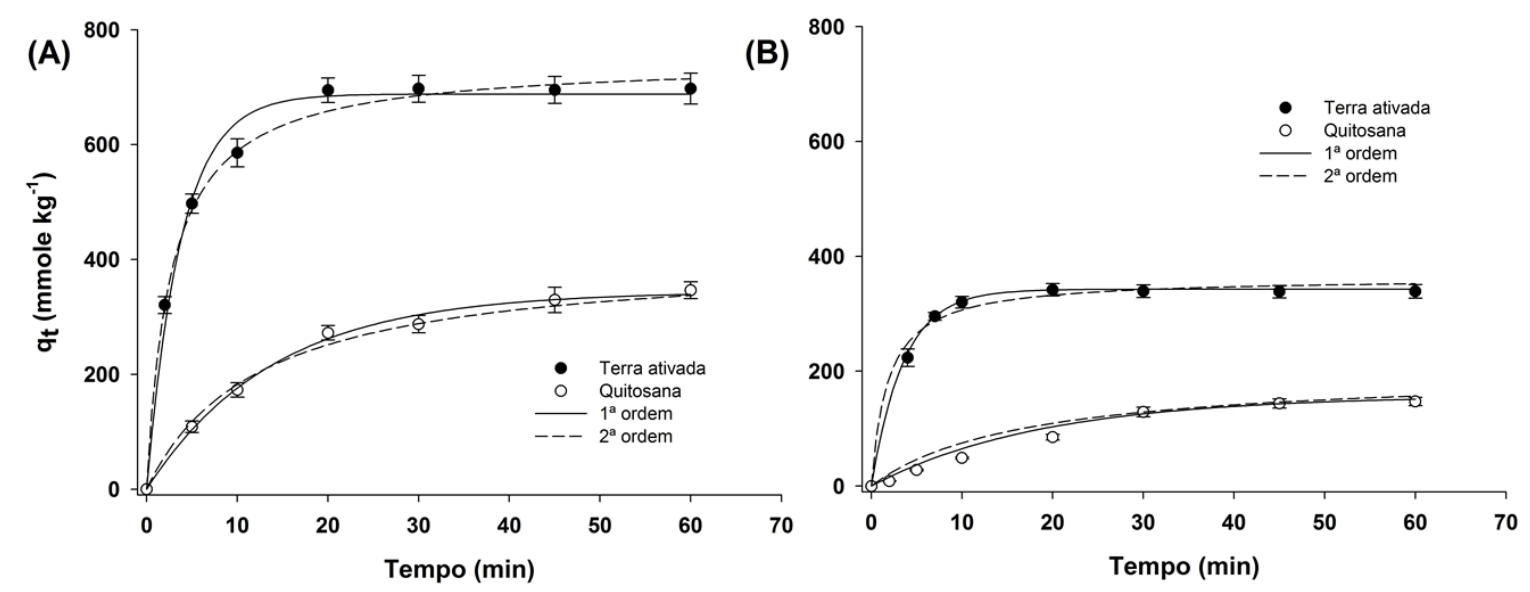

\subsection{Avaliação dos modelos cinéticos}

Ambos os modelos de pseudo-primeira ordem e pseudo-segunda ordem descreveram satisfatoriamente o comportamento cinético da adsorção de carotenóides por terra ativada (Tabela 1), comprovado pelos altos valores do coeficiente de determinação ajustado $\left(\mathrm{R}_{\text {aju. }}^{2}>\right.$ $0,99)$. Porém, o modelo de pseudo-segunda ordem apresentou menor erro médio relativo $($ EMR $<5 \%$ ), independente da quantidade de adsorvente. O mesmo comportamento foi observado para a adsorção de peróxidos, especialmente utilizando $1 \%$ de terra ativada. A constante da taxa de adsorção $\left(k_{1}\right)$ está relacionada com a velocidade do processo de adsorção. Assim, utilizando $2 \%$ de terra ativada, carotenóides e peróxidos foram adsorvidos mais rapidamente do que com $1 \%$ de adsorvente. A mesma tendência foi notada por Silva et al. 
(2013) estudando a cinética de branqueamento de óleo de palma com terra ativada (Tonsil OPT $210 \mathrm{FF})$.

Tabela 1 - Parâmetros cinéticos da adsorção de carotenóides e peróxidos de OFA

\begin{tabular}{|c|c|c|c|c|}
\hline \multirow{2}{*}{$\begin{array}{l}\text { Adsorbato } \\
\text { Adsorvente }\end{array}$} & \multicolumn{2}{|l|}{ Carotenóides } & \multicolumn{2}{|l|}{ Peróxidos } \\
\hline & Terra ativada & Quitosana & Terra ativada & Quitosana \\
\hline \multicolumn{5}{|c|}{$1 \%$ de adsorvente } \\
\hline \multicolumn{5}{|c|}{ Pseudo-primeira ordem } \\
\hline $\mathrm{q}_{1}\left(\mathrm{mg} \mathrm{kg}^{-1}\right)$ & $658,28 \pm 19,52$ & $183,30 \pm 16,74$ & $687,90 \pm 7,97$ & $343,85 \pm 3,66$ \\
\hline $\mathrm{k}_{1}\left(\min ^{-1}\right)$ & $0,1889 \pm 0,0030$ & $0,0690 \pm 0,0107$ & $0,2642 \pm 0,0087$ & $0,0717 \pm 0,0002$ \\
\hline $\mathrm{R}^{2}$ & 0,992 & 0,975 & 0,989 & 0,995 \\
\hline $\mathrm{R}_{\text {aiu. }}^{2}$ & 0,990 & 0,970 & 0,987 & 0,994 \\
\hline $\operatorname{EMR}(\%)$ & 2,89 & 7,29 & 3,78 & 2,61 \\
\hline \multicolumn{5}{|c|}{ Pseudo-segunda ordem } \\
\hline $\mathrm{q}_{2}\left(\mathrm{mg} \mathrm{kg}^{-1}\right)$ & $718,45 \pm 20,01$ & $229,91 \pm 13,28$ & $746,44 \pm 9,11$ & $406,48 \pm 6,83$ \\
\hline $\mathrm{k}_{2}\left(\mathrm{~kg} \mathrm{mg}^{-1} \mathrm{~min}^{-1}\right)$ & $0,004 \pm 0,0001$ & $0,0003 \pm 0,0000$ & $0,0005 \pm 0,0001$ & $0,0002 \pm 0,00001$ \\
\hline $\mathrm{R}^{2}$ & 0,998 & 0,971 & 0,996 & 0,993 \\
\hline $\mathrm{R}_{\text {aiu. }}^{2}$ & 0,998 & 0,965 & 0,995 & 0,991 \\
\hline $\operatorname{EMR}(\%)$ & 2,01 & 9,07 & 1,72 & 4,3 \\
\hline \multicolumn{5}{|c|}{$2 \%$ de adsorvente } \\
\hline \multicolumn{5}{|c|}{ Pseudo-primeira ordem } \\
\hline $\mathrm{q}_{1}\left(\mathrm{mg} \mathrm{kg}^{-1}\right)$ & $535,02 \pm 14,29$ & $153,87 \pm 9,37$ & $342,63 \pm 5,00$ & $170,99 \pm 2,23$ \\
\hline $\mathrm{k}_{1}\left(\min ^{-1}\right)$ & $0,3197 \pm 0,0002$ & $0,0604 \pm 0,0088$ & $0,2817 \pm 0,0053$ & $0,0381 \pm 0,0012$ \\
\hline $\mathrm{R}^{2}$ & 0,991 & 0,997 & 0,996 & 0,989 \\
\hline $\mathrm{R}^{2}$ aiu. & 0,989 & 0,996 & 0,995 & 0,986 \\
\hline $\operatorname{EMR}(\%)$ & 2,70 & 5,42 & 1,53 & 10,86 \\
\hline \multicolumn{5}{|c|}{ Pseudo-segunda ordem } \\
\hline $\mathrm{q}_{2}\left(\mathrm{mg} \mathrm{kg}^{-1}\right)$ & $565,21 \pm 15,10$ & $189,29 \pm 9,45$ & $362,92 \pm 4,53$ & $223,52 \pm 6,20$ \\
\hline $\mathrm{k}_{2}\left(\mathrm{~kg} \mathrm{mg}^{-1} \mathrm{~min}^{-1}\right)$ & $0,0011 \pm 0,0001$ & $0,0003 \pm 0,0000$ & $0,0015 \pm 0,0000$ & $0,0002 \pm 0,00000$ \\
\hline $\mathrm{R}^{2}$ & 0,999 & 0,992 & 0,979 & 0,982 \\
\hline $\mathrm{R}_{\text {aju. }}^{2}$ & 0,998 & 0,991 & 0,974 & 0,978 \\
\hline EMR (\%) & 0,96 & 10,03 & 4,26 & 16,45 \\
\hline
\end{tabular}

$\mathrm{O}$ modelo de pseudo-primeira ordem foi mais adequado para representar o comportamento cinético do branqueamento de OFA, utilizando quitosana como adsorvente. A quitosana apresentou uma constante da taxa de adsorção $\left(k_{l}\right)$ e uma capacidade de adsorção $(q)$ inferior à terra ativa em todos os experimentos, o que dificulta seu uso na forma de pó como adsorvente para o branqueamento de óleo de arroz. Porém, visto que a quitosana possui grupos reativos, modificações na sua estrutura que possibilitem aumentar a área superficial ou alterar o grau de desacetilação do polímero podem melhorar a capacidade de adsorção e tornar viável a sua utilização no branqueamento, especialmente para remoção de produtos de oxidação, visto que a permanência de carotenóides após o branqueamento pode ser vantajoso por retardar o processo de oxidação e aumentar a qualidade nutricional de OFA. 


\section{CONCLUSÃO}

A adsorção de carotenóides e peróxidos por terra ativada e quitosana foi estudada na etapa de branqueamento de óleo de farelo de arroz (OFA). A terra ativada demonstrou alta capacidade de adsorção de carotenóides e peróxidos. A quitosana manteve uma grande quantidade de carotenóides no óleo e reduziu quase $40 \%$ dos peróxidos, o que dificulta sua utilização no branqueamento de OFA, sem modificações na sua estrutura. O modelo de pseudo-segunda ordem foi mais adequado para descrever o processo de branqueamento com terra ativada e o modelo de pseudo-primeira ordem se ajustou melhor aos experimentos com quitosana.

\section{REFERÊNCIAS}

AOCS. Official and tentative methods of the American Oil Chemist's Society. Chicago, USA: American Oil Chemists' Society Press, 1998.

MOURA, C. M.; MOURA, J. M.; SOARES, N. M.; PINTO, L. A. A. Evaluation of molar weight and deacetylation degree of chitosan during chitin deacetylation reaction: used to produce biofilm. Chem. Eng. Process., v. 50, p. 351-355, 2011.

MPOB Test Method. Determination of Carotene Content. Malaysian Palm Oil Board, Method $\mathrm{n}^{\mathrm{o}}$. p 2.6, p. 194-197, 2005.

PESTANA-BAUER, V. R.; ZAMBIAZI, R. C.; MENDONÇA, C. R. B.; BENEITOCAMBRA, M.; RAMIS-RAMOS, G. y-oryzanol and tocopherol contents in residues of rice bran oil refining. Food Chem., v. 134, p. 1479-1483, 2012.

SATHIVEL, S. PRINYAWIWATKUL, W. Adsorption of FFA in Crude Catfish Oil onto Chitosan, Activated Carbon, and Activated Earth: A Kinetics Study. J. Am. Oil. Chem. Soc., v. 81, n. 4, 2004.

SILVA, S. M.; SAMPAIO, K. A.; CERIANI, R.; VERHÉ, R. STEVENS, C.; DE GREYT, W.; MEIRELLES, A. J. A. Effect of type of bleaching earth on the final color of refined palm oil. Lebensm. Wiss. Technol., v. 59, p. 1258-1264, 2014.

SILVA, S. M.; SAMPAIO, K. A.; CERIANI, R.; VERHÉ, R.; STEVENS, C.; DE GREYT, W.; MEIRELLES, A. J. A. Adsorption of carotenes and phosphorus from palm oil onto acid activated bleaching earth: Equilibrium, kinetics and thermodynamics. J. Food Eng., v. 118, p. 341-349, 2013.

WAN NGAH, W. S.; TEONG, L. C.; HANAfiAH, M. A. K. M. Adsorption of dyes and heavy metal ions by chitosan composites: A review. Carbohyd. Pol., v. 83, p. 14461456, 2011.

WORASITH, N.; GOODMAN, B. A.; JEYASHOKE, N.; THIRAVETYAN, P. Decolorization of Rice Bran Oil Using Modified Kaolin. J. Am. Oil. Chem. Soc., v. 88, p. 2005-2014, 2011. 